\title{
Requests of the cosmic ray community to accelerator high energy physicists
}

\author{
Lawrence W. Jones $^{\mathrm{a}}$ \\ University of Michigan, Ann Arbor, Michigan, USA
}

Abstract. The requests of Cosmic Ray physicists to Accelerator physicists are summarized.

\section{Introduction, current situation and future}

This was a topic of discussion at the last three ISVHECRI meetings at Fermilab, in Berlin and at CERN respectively. Indeed, the cosmic ray request is still relevant. The issue is the understanding of cosmic ray air showers.

At and above PeV energies, most cosmic ray data is based on ground-level observations of air showers, which are the results of the interactions of primary cosmic rays with air nuclei. The observables, on which the energy and particle nature of the primary cosmic ray are based, are mostly electrons and muons, plus some hadrons. Of course, at these $\mathrm{PeV}$ energies $\left(>10^{15} \mathrm{eV}\right)$, there is no direct accelerator data on the collision of protons with air nuclei. However, colliding beam data, where a proton of $7.5 \mathrm{TeV}$ collides with a nucleus of oxygen $\left(\mathrm{O}^{16}\right)$ of about $60 \mathrm{TeV}$ (i.e., the same magnetic rigidity as a $7.5 \mathrm{TeV}$ proton) would produce a final state distribution of observable particles which would be directly observed and studied. Such a collision would be equivalent to a proton of about a PeV colliding with a stationary oxygen nucleus.
The LHC has had runs with collisions of lead nuclei with other lead nuclei, and also with collisions of protons with lead nuclei, so colliding beams of heavy nuclei or collisions between protons and other nuclei are altogether practical. What is needed is an agreement with the leadership of at least one of the major collider experiments to collaborate in a proton-oxygen (or protonnitrogen) colliding beam program. Probably only a week of data would be needed, to produce enough data to finetune the existing cosmic ray air-shower models. Perhaps some runs with collisions between light nuclei (e.g., He, $\mathrm{Be}, \mathrm{C}$, etc.) would also be useful. What is most needed is a close communication between active and involved members of the cosmic ray community (e.g., participants in the understanding of AUGER data) and members of at least one of the LHC experiments; ATLAS or CMS, for example.

It is hoped that agreement can be found to include a proton-oxygen run in the next program devoted to heavyion collisions.

\footnotetext{
a e-mail: 1wjones@umich.edu

(C) The Authors, published by EDP Sciences. This is an Open Access article distributed under the terms of the Creative Commons Attribution License 4.0
} (http://creativecommons.org/licenses/by/4.0/). 